\title{
FORMATION OF DIFFUSION WAVES IN A SCALAR CONSERVATION LAW WITH CONVECTION
}

\author{
KEVIN R. ZUMBRUN
}

\begin{abstract}
We study the scalar conservation law, $u_{t}+[c(x) u]_{x}+b\left(u^{2}\right)_{x}=u_{x x}$, $c^{\prime} \geq 0$, which is a model equation for the behavior of weak transverse waves near a viscous transitional shock. Solutions are shown to decay in $L^{1}$ to a pair of diffusion waves, moving apart at speeds $c(-\infty)$ and $c(+\infty)$, behavior that has been observed numerically in solutions of the full equations. The interesting aspect of the analysis is that the asymptotic state of the solution is not known a priori, in contrast to cases treated previously.
\end{abstract}

\section{INTRODUCTION}

We study the scalar conservation law

$$
u_{t}+[c(x) u]_{x}+b\left(u^{2}\right)_{x}=u_{x x}
$$

where $c(x)$ is a nondecreasing function such that $c^{\prime}(x)$ and $c^{\prime \prime}(x)$ decay exponentially at infinity $c(-\infty)<0<c(+\infty)$ and $c^{2}+c^{\prime} \not \equiv 0$ (a technical condition). The constant $b$ is arbitrary.

This equation describes the behavior of nonlinear waves moving through a convection field $c(x)$. With the choice of $c(x)=\tanh (x)$, it serves as a model equation for the behavior of weak transverse waves near a transitional shcok wave; here $c(x)$ represents the convection induced by the underlying shock and cross-terms are ignored (cf. $[8,10])$.

Our interest is in understanding the behavior at the level of diffusion waves. For weak classical shocks, Liu [7] has shown that the large-time behavior of transverse waves is to coalesce into a single diffusion wave leaving the shock. This diffusion wave is either a standard heat kernel or the analogous source solution of Burgers' equation, according to whether the associated characteristic family is linearly degenerate or genuinely nonlinear. Numerical experiments [10] suggest that transverse waves near transitional shock exhibit analogous behavior. However, since transverse waves leave the shock on either side, the asymptotic state consists of two emerging diffusion waves.

Received by the editors November 12,1991; originally communicated to the Proceedings of the $A M S$ by James G. Glimm.

1991 Mathematics Subject Classification. Primary 35B35, 35B40, 35L65, 35L67, 35L80.

Key words and phrases. Conservation laws, non-strictly-hyperbolic, transitional shock waves, convection, diffusion waves.

This work was supported in part by the National Science Foundation Postdoctoral Program under Grant DMS-9107990 and the National Science Foundational under Grant DMS-8901884. 
Likewise, in (1.1), mass is convected in the separated directions $\frac{d x}{d t}=c(-\infty)$ and $\frac{d x}{d t}=c(+\infty)$. The corresponding result is:

Theorem 1 (Convergence to diffusion waves). Let $u$ be a solution of Eq. (1.1) with initial data $u_{0} \in C^{3}$ such that $u_{0}, u_{0_{x}}$, and $u_{0_{x x}}$ decay exponentially at infinity and $\left\|u_{0}\right\|_{L^{\infty}}$ is sufficiently small. Then, for any choice of $\varepsilon>0, u$ satisfies

$$
\|u-\Theta\|_{L^{1}}=O(1) t^{-1 / 2+\varepsilon}
$$

where

$$
\Theta=\theta_{1}(x-c(-\infty) t, t)+\theta_{2}(x-c(+\infty) t, t)
$$

and $\theta_{1}$ and $\theta_{2}$ are source solutions of

$$
\theta_{t}+b(\theta)_{x}^{2}=\theta_{x x}
$$

i.e. solutions with $\theta_{i}(x, 0)=\rho_{i} \delta(x)$, where $\delta(x)$ is the dirac delta function.

This result is proved in the following sections. We remark that it is sufficient to assume $u_{0}$ merely small in $\|\cdot\|_{L^{\infty}}$ and exponentially decaying at infinity, as the remaining hypotheses must then hold for any sufficiently small $t>0$. We will not prove this last claim, which belongs to the short-time theory of general second-order parabolic equations (cf. [3, 4]).

The analysis of the classical case is facilitated by prior knowledge of the asymptotic state; conservation of mass is enough to describe completely the single emerging diffusion wave [7]. In (1.1), conservation of mass gives the total mass of the two emerging diffusion waves but does not determine the individual mass of either. Thus the standard analysis is precluded here.

Our alternate approach proceeds in two steps. We first establish exponential decay of the solution and its derivative in an approximate weighted norm, similar to that introduced in [9] for the study of linearized stability of shock waves. This implies exponential decay in the standard norm on any bounded set; in particular, it implies expoential decay of the mass flux through $x=0$. Thus, the solution separates into left and right components moving away from the origin.

Next, we observe that, since $c(x)$ decays rapidly to a constant at plus and minus infinity, the two portions of the solution approximately satisfy constantconvection equations with different speeds, equations which fall under the standard theory. By comparison with the exact solutions of these equations, we establish their convergence to diffusion waves.

As remarked in [10], it is a common feature of problems related to transitional waves that the asymptotic state of solutions is not known a priori. Our treatment of (1.1) should be viewed as a first attempt in developing analytic methods for their study.

\section{SOURCE SOlutions}

A source solution $\theta(x, t)$ of the scalar equation

$$
u_{t}+b u_{x}^{2}=u_{x x}
$$

is a solution with initial data

$$
\theta(x, 0)=\rho \delta(x),
$$


where $\delta(x)$ is the dirac delta function. We will use the notion $\theta(x, t ; \rho)$ when we wish to indicate a specific source solution.

In the case $b=0, \theta$ is simply a heat kernel

$$
\theta(x, t ; \rho)=(\rho / \sqrt{4 \pi t}) e^{-x^{2} / 4 t} .
$$

In the case $b \neq 0$, the Hopf-Cole transformation

$$
u=-(1 / b)\left(w_{x} / w\right)
$$

reduces $(2.1)$ to the heat equation $w_{t}=w_{x x}$, whereby we obtain

$$
\theta(x, t ; \rho)=\frac{(1 / b \sqrt{4 \pi t}) e^{-x^{2} / 4 t}}{c \tanh (\rho b / 2)-\operatorname{erf}(x / \sqrt{4} \pi t)} .
$$

Proposition 2.1. Let $u(x, t)$ be a solution of

$$
u_{t}+b\left(u^{2}\right)_{x}=u_{x x}
$$

with initial data $u_{0}(x)$, and let $\rho=\int_{-\infty}^{+\infty} u_{0}(x) d x$. Then

$$
\|u(x, t)-\theta(x, t ; \rho)\|_{L^{1}}=O(1) t^{-1 / 2}\left\|x u_{0}\right\|_{L^{1}} .
$$

Proof. We treat only the case $b=0$. The case $b \neq 0$ follows similarly, using the Hopf-Cole transformation.

By the explicit solution of the heat equation, the function $h=u-\theta$ is given by

$$
h(x, t)=h(x, 0) * K(x, t)=-H(x, 0) * K_{x}(x, t),
$$

where $*$ denotes convolution, $K(x, t)=(1 / \sqrt{4 \pi t}) e^{-x^{2} / 4 t}$ is the heat kernel, and $H(x, t)=\int_{-\infty}^{x} h(x) d x$. Thus,

$$
\|h\|_{L^{1}} \leq\|H(x, 0)\|_{L^{1}}\left\|K_{x}(x, t)\right\|_{L^{1}}=t^{-1 / 2}\|H(x, 0)\|_{L^{1}} .
$$

But $\int_{-\infty}^{+\infty} h(x) d x=0$, so that $\|H(x, 0)\|_{L^{1}} \leq\|x h(x, 0)\|_{L^{1}}$ (integration by parts). Observing that $\|x h(x, 0)\|_{L^{1}}=\|x u(x, 0)-x \rho \delta(x)\|_{L^{1}}=\|x u(x, 0)\|_{L^{1}}$, we are done.

Corollary 2.2. Let $u(x, t)$ be a solution of

$$
u_{t}+c u_{x}+b\left(u^{2}\right)_{x}=u_{x x}
$$

on $t \geq T$, for $c$ constant; and let $\rho=\int_{-\infty}^{+\infty} u(x, T) d x$. Then

$$
\|u(x, t)-\theta(x-c, t ; \rho)\|_{L^{1}}=O(1)(t-T)^{-1 / 2}\|(x-c T) u(x, T)\|_{L^{1}} .
$$

Proof. The change of coordinates $\left(x^{\prime}, t^{\prime}\right)=(x-c t, t-T)$ reduces this to the previous proposition.

\section{COMPARISON THEOREMS}

The main tool required in our analysis is an $L^{1}$ stability estimate familiar from hyperbolic theory [5]: 
Proposition 3.1 ( $L^{1}$ stability). For $T_{0} \leq t \leq T_{1}$, let $u^{1}, u^{2} \in C^{2}$ be solutions of

$$
u_{t}^{i}+f\left(u^{i}, x, t\right)_{x}=\alpha(x, t) u^{i}+u_{x x}^{i}+S^{i}(x, t),
$$

where $f$ is smooth, $\left|f_{u}\right|,|\alpha|,\left\|S^{1}-S^{2}\right\|_{L^{1}}$, and $\left\|u^{1}-u^{2}\right\|_{L^{1}}$ are bounded, and $\left|u^{1}-u^{2}\right|,\left|u_{x}^{1}-u_{x}^{2}\right| \rightarrow 0$ as $x \rightarrow \pm \infty$, uniformly in $t$. Then

$$
\left\|u^{1}-u^{2}\right\|_{L^{1}}\left(T_{1}\right)-\left\|u^{1}-u^{2}\right\|_{L^{1}}\left(T_{0}\right) \leq \int_{T_{0}}^{T_{1}} \int_{-\infty}^{+\infty} \alpha\left|u^{1}-u^{2}\right| d x d t+\int_{T_{0}}^{T_{1}}\left\|S^{1}-S^{2}\right\|_{L^{1}} d t .
$$

Proof. Subtract the equations for $u^{1}$ and $u^{2}$ given by (2.1), multiply by $\operatorname{sgn}\left(u^{1}-u^{2}\right)$, and integrate over the strip $T_{0} \leq t \leq T_{1}$. Applying Green's theorem to the terms involving derivatives, we find that, at the curves $u^{1}=u^{2}$, the boundary terms corresponding to the flux $f$ cancel, while those corresponding to the dissipation terms $u_{x x}$ have the correct sign. Boundary terms at $x= \pm \infty$ vanish by our assumptions on $\left|u^{1}-u^{2}\right|$ and on $f$.

We also make use of the following comparision principles:

Proposition 3.2 (Monotonicity). Let $u^{1}, u^{2} \in C^{2}$ be solutions of

$$
u_{t}^{i}+f\left(u^{i}, x, t\right)_{x}=\alpha(x, t) u^{i}+u_{x x}^{i}+S^{i}(x, t),
$$

where $f \in C^{2}, f_{u x}$ and $\alpha$ are bounded, and $S^{1} \geq S^{2}$. If the initial data satisfy $u_{0}^{1} \geq u_{0}^{2}$, then $u^{1} \geq u^{2}$ for all time.

Proof. Define

$$
\tilde{f}(x, t)=\left[f\left(u^{2}, x, t\right)-f\left(u^{1}, x, t\right)\right]\left(u^{2}-u^{1}\right)^{-1}
$$

and $\omega\left(u^{2}-u^{1}\right) e^{-K t}$, so that

$$
\omega_{t} / l e\left(\tilde{f}_{x}-K+\alpha\right) \omega+\tilde{f} \omega_{x}+\omega_{x x} .
$$

The function $\tilde{f}_{x}$ is bounded on $t \leq T$ by $\max _{t \leq T} f_{u x}$. Choosing

$$
K>\max _{t \leq T}\left(\tilde{f}_{x}+\alpha\right),
$$

$\omega$ satisfies a maximum principle on $t \leq T$ so that $\omega$, and therefore $u^{2}-u^{1}$, is nonpositive on $t \leq T$. Since $T$ is arbitrary, the result follows.

Proposition 3.3. Let $u^{1}, u^{2}>0$ satisfy

$$
u_{t}^{i}+\left[c^{i}(x, t) u^{i}\right]_{x}=u_{x x}^{i},
$$

where $c^{1} \geq c^{2}$ and $u_{0}^{1} \geq u_{0}^{2}$. Then, for any $a$,

$$
\int_{a}^{+\infty} u^{1} d x \geq \int_{a}^{+\infty} u^{2} d x
$$

for all time.

Proof. This is suggested by the interpretation of $(3.5)$ as diffusion processes, with drift velocities $c^{i}$. Setting $W(x, t)=\int_{x}^{+\infty}\left(u^{2}(x, t)-u^{1}(x, t)\right) d x$, we have

$$
W_{t}+c^{1}(x, t) W_{x}=W_{x x}-u^{2}\left(c^{1}-c^{2}\right)
$$


By hypothesis, $-u^{2}\left(c^{1}-c^{2}\right) \leq 0$, so that $W$ satisfies a maximum principle.

Remark. The properties of $L^{1}$ contraction and monotonicity are equivalent when local existence holds (cf. [5]).

\section{Separation of the solution}

In what follows, we may take $u \in C^{3}$ to be a global solution of (1.1) for which $u, u_{x}$, and $u_{x x}$ decay at $x= \pm \infty$. This follows from local existence theory (cf. $[2,3,4])$ up to a time depending only on $\left\|u_{0}\right\|_{L^{\infty}}$. Since $\|u\|_{L^{\infty}}$ is uniformly bounded, by the maximum principle, the global result follows from repeated application of the short time theory. From our assumptions on $u$, it follows that all functions defined below satisfy the hypothesis of Proposition 3.1.

Proposition 4.1. The solution $u(x, t)$ of (1.1) decays in $\|\cdot\|_{L_{\infty}}$ and $\|\cdot\|_{L_{1}}$. Proof. This follows from the maximum prinicple and from Proposition 3.1 and $u^{1}=u$ and $u^{2} \equiv 0$.

Now we define $v(x, t)$ by

$$
u(x, t)=\Lambda(x) v(x, t),
$$

where $\Lambda(x)$ satisfies $\Lambda_{x}=\eta c \Lambda, \Lambda(0)=1$, and $0<\eta<1$. In terms of $v$, (1.1) becomes

$$
v_{t}=v_{x x}-\left[(1-\eta) c^{\prime}+\eta(1-\eta) c^{2}+2 \eta b c u\right] v-[(1-2 \eta) c+2 b u] v_{x} .
$$

Proposition 4.2. $\|v\|_{L^{\infty}},\|v\|_{L_{1}}$, and $\left\|v_{x}\right\|_{L_{1}}$ decay exponentially, provided that $\left\|u_{0}\right\|_{L^{\infty}}$ is sufficiently small.

Proof. The quantity $-\left[(1-\eta) c^{\prime}+\eta(1-\eta) c^{2}\right]$ is negative and bounded away from zero. Thus, in (4.2), the coefficient of the source term satisfies

$$
-\left[(1-\eta) c^{\prime}+\eta(1-\eta) c^{2}+2 \eta b c u\right] \leq-\sigma<0,
$$

as long as $\|u(\cdot, 0)\|_{L^{\infty}}$ is sufficiently small. Applying the maximum principle, we obtain $L^{\infty}$ decay in $v$ at the rate of $e^{-\sigma t}$.

Rearranging (4.2), we obtain

$$
v_{t}+([(1-2 \eta) c+b u] v)_{x}=-\left[\eta c^{\prime}+\eta(1-\eta) c^{2}+\eta b c u\right] v+v_{x x},
$$

where, again, the coefficient of the source term is uniformly negative, say, less than $-\tilde{\sigma}<0$. Applying Proposition 3.1 with $u^{1}=v, u^{2} \equiv 0$, we obtain the Gronwall inequality

$$
\frac{d}{d t}\|v\|_{L^{1}} \leq-\tilde{\sigma}\|v\|_{L^{1}}
$$

giving $L^{1}$ decay in $v$ at the rate $e^{-\tilde{\sigma} t}$.

Now set $\omega=v_{x}$. Writing (4.2) as

$$
v_{t}=v_{x x}-\alpha(x, t) v+\beta(x, t) v_{x}
$$

and differentiating gives

$$
\omega_{t}-[\beta \omega]_{x}=\omega_{x x}-\alpha \omega-\alpha_{x} v .
$$


The term $\alpha_{x} v$ may be split into $O(1) v+O(1) u_{x} v=O(1) v+O(1) \omega u$ to give

$$
\omega_{t}-[\beta \omega]_{x}=\omega_{x x}-[\alpha+O(1) u] \omega+O(1) v,
$$

where, again, $-[\alpha+O(1) u] \leq-\bar{\sigma}<0$, provided that $\left\|u^{0}\right\|_{L^{\infty}}$ is sufficiently small.

Applying Proposition 3.1 with $u^{1}=\omega, u^{2} \equiv 0$, and using $\left\|S^{1}-S^{2}\right\|_{L^{1}}=$ $O(1)\|v\|_{L^{1}}=O(1) e^{-\tilde{\sigma}(t)}$, we obtain the Gronwall inequality

$$
\frac{d}{d t}\|\omega\|_{L^{1}} \leq-\bar{\sigma}\|\omega\|_{L^{1}}+O(1) e^{-\tilde{\sigma}(t)}
$$

which implies that $\|\omega\|_{L^{1}}=O(1) e^{-(\min \{\bar{\sigma}, \tilde{\sigma}\}-\varepsilon) t}$, for any $\varepsilon>0$.

Corollary 4.3. $\|u\|_{L^{\infty}},\|u\|_{L^{1}}$, and $\left\|u_{x}\right\|_{L^{1}}$ decay exponentially on any bounded set.

Remarks. (1) The results of this section require only that $c^{2}+c^{\prime}$ be bounded away from zero. In particular, this allows for $c$ to be a nonzero constant, which models behavior of transverse waves in the presence of a classical shock.

(2) When $c(x)$ is taken to be a nonzero constant, the choice $\eta=\frac{1}{2}$ gives sharp decay information. This is one way to estimate "almost finite" propagation speed in the heat equation and Burgers' equation.

(3) The uncontrolled term $u_{x} \omega$ contained in the $[\beta \omega]_{x}$ term in (4.6), prevents us from obtaining decay in $\|\omega\|_{L^{\infty}}$ by the maximum principle. This is familiar from the example of Burgers' equation, where our argument again gives decay in $\left\|u_{x}\right\|_{L^{\prime}}$ but not in $\left\|u_{x}\right\|_{L^{\infty}}$. This simply reflects the fact that the total variation norm decreases, even though the gradient may grow large at certain points.

\section{PROOF OF THE MAIN THEOREM}

We now investigate the formation of diffusion waves. Without loss of generality, we consider only the behavior on $x \geq 0$. By choosing $\eta$ sufficiently small, we can assume that $[c(x)-c(+\infty)]$ and $c^{\prime}(x)$ decay faster at infinity than $\Lambda^{-1}=O(1) e^{-\eta c(+\infty)|x|}$. Therefore we can rewrite (1.1) as

$$
u_{t}+c(+\infty) u_{x}+b\left(u^{2}\right)_{x}=u_{x x}-S(x, t)
$$

where the source term $S(x, t)=[(c-c(+\infty)) u]_{x}$ is bounded in absolute value by $O(1)\left(|v|+\left|v_{x}\right|\right)$ and thus decays in $\|\cdot\|_{L^{1}}$ as $e^{-\sigma t}$ for some $\sigma>0$.

For $t \geq T$, we define $\bar{u}(x, t ; T)$ to be the solution of

$$
\bar{u}_{t}+c(+\infty) \bar{u}_{x}+b\left(\bar{u}^{2}\right)_{x}=\bar{u}_{x x}
$$

with initial data $\bar{u}(x, T ; T)$ equal to $u(x, T)$ on $x \geq 0$ and zero elsewhere.

By mimicking the proof of Proposition 3.1, we obtain an estimate

$$
\frac{d}{d t}\left\|u^{1}-u^{2}\right\|_{L^{1}[a, \infty]} \leq \int_{a}^{+\infty} \alpha\left|u^{1}-u^{2}\right| d x+\left\|S^{1}-S^{2}\right\|_{L^{1}[a, \infty]}+\Gamma(a, t)
$$

containing the additional boundary term

$$
\Gamma(a, t)=\left|f\left(u^{1}(a, t), a, t\right)-f\left(u^{2}(a, t), a, t\right)\right|+\left|u_{x}^{1}(a, t)-u_{x}^{2}(a, t)\right| .
$$

Setting $u^{1}=u$ and $u^{2}=\bar{u}$, we have

$$
\Gamma=\left|c(+\infty) u+b u^{2}-c(+\infty) \bar{u}-b \bar{u}^{2}\right|+\left|u_{x}-\bar{u}_{x}\right|=O(1)\left(|u|+\left|u_{x}\right|\right) .
$$


Let $l>0$ be fixed. Since Corollary 4.3 applies to $\bar{u} a$ by Remark (1) of $\S 4$ and to $u$ considered as a solution of the original equation (1.1), we have $\|\Gamma(x, t)\|_{L^{1}[0, l]}=O(1) e^{-\hat{\sigma} t}$, for some $\hat{\sigma}>0$, so that

$$
\int_{T}^{\infty} \int_{0}^{t}|\Gamma(x, t)| d x d t=O(t) e^{-\hat{\sigma} T}
$$

It follows that there is some $a \in[0, l]$ for which

$$
\int_{T}^{\infty}|\Gamma(a, t)| d t=(1 / l) O(1) e^{-\hat{\sigma} T} .
$$

Applying (5.3) and noting that $\alpha \equiv 0,\|u(x, T)-\bar{u}(x, T ; T)\|_{L^{1}[a, \infty]}=0$, and $\left|S^{1}-S^{2}\right|_{L^{1}}=O(1) e^{-\sigma t}$ gives

$$
\| u(x, t)-\bar{u}\left(x, t ; T \|_{L^{1}[a, \infty]}(T) \leq O(1) e^{-\tilde{\sigma} T},\right.
$$

for all $t \geq T$, where $\tilde{\sigma}=\min \{\hat{\sigma}, \sigma\}$. Therefore, we have

$$
\|u-\bar{u}\|_{L^{1}[0, \infty]}, \quad(T) \leq O(1) e^{-\tilde{\sigma} T},
$$

for $t \geq T$, as well, since $\|u\|_{L^{1}[0, a]}$ and $\|\bar{u}\|_{L^{1}[0, a]}$ are $O(1) e^{-\sigma T}$, as noted above.

By Corollary 2.2, $\bar{u}$ converges to a diffusion wave as $t \rightarrow \infty$. To estimate the rate of this convergence, we introduce $\overline{\bar{u}}(x, t ; T)$ satisfying (5.2), with initial data $\overline{\bar{u}}(x, T ; T)$ equal to $u(x, T)$ on $[0, C T]$ and zero elswhere.

Lemma 5.1. For $C$ sufficiently large, $\|u(x, T)\|_{L^{1}[C T, \infty]}=O(1) e^{-\sigma T}$, for $\sigma>$ 0 .

Proof. Let $\tilde{u}$ be the solution of the constant-convection equation (5.10)

$$
\tilde{u}+c_{\max } \tilde{u}=\tilde{u} x x,
$$

with initial data $\tilde{u}^{0}=\left|u_{0}\right|$ and $c_{\max }=\max |c(x)+b u|$. By Propositions 3.2 and 3.3, $\|u\|_{L^{1}[C t, \infty]}$ is bounded by $\|\tilde{u}\|_{L^{1}[C t, \infty]}$.

But, for $C>c_{\max },\|\tilde{u}\|_{L^{1}[C t, \infty]}$ decays exponentially, a property of "almost finite" speed of propagation that is well known for the constant-convection equation (5.10). We can verify this by the change of coordinates $\left(x^{\prime}, t^{\prime}\right)=$ $(x-C t, t)$, together with the observations in Remarks (1) and (2) of $\S 4$.

Lemma 5.1 implies that $\|\overline{\bar{u}}(x, t ; T)-\overline{\bar{u}}(x, T ; T)\|_{L^{1}}=O(1) e^{-\sigma T}$. Thus, by Proposition 3.1,

$$
\|\overline{\bar{u}}(x, t ; T)-\bar{u}(x, t ; T)\|_{L^{1}}=O(1) e^{\sigma T}
$$

for all $t \geq T$. But, since

$$
\|(x-c(+\infty) T) \overline{\bar{u}}(x, T ; T)\|_{L^{1}} \leq C T\|u(x, T)\|_{L^{\infty}},
$$

Corollary 2.2 gives

$$
\left\|\overline{\bar{u}}(x, t ; T)-\theta\left(x-c(+\infty) t, t ; \rho_{T}\right)\right\|_{L^{1}}=O(1) T(t-T)^{-1 / 2},
$$

with $\rho_{T} \equiv \int_{0}^{C T} u(x, t) d x$. Combining (5.9), (5.12), and (5.13), we have

(5.14) $\left\|u(x, t)-\theta\left(x-c(+\infty) t, t ; \rho_{T}\right)\right\|_{L^{1}[0, \infty]}=O(1)\left(e^{-\bar{\sigma} T}+T(t-T)^{-1 / 2}\right)$,

for $t \geq T$, where $\bar{\sigma}=\min \{\tilde{\sigma}, \sigma\}$. 
From (5.14), we obtain

$$
\begin{aligned}
& \left\|\theta\left(x-c(+\infty) t, t ; \rho_{T_{1}}\right)-\theta\left(x-c(+\infty) t, t ; \rho_{T_{2}}\right)\right\|_{L^{1}[0, \infty]} \\
& \quad=O(1)\left(e^{-\bar{\sigma} T}+T(t-T)^{-1 / 2}\right)
\end{aligned}
$$

for $t \geq T$, where $T=\max \left\{T_{1}, T_{2}\right\}$. It is clear that

$$
\left\|\theta\left(x-c(+\infty) t, t ; \rho_{1}\right)-\theta\left(x-c(+\infty) t, t ; \rho_{2}\right)\right\|_{L^{1}}=\left|\rho_{1}-\rho_{2}\right|,
$$

so that

$$
\left\|\theta\left(x-c(+\infty) t, t ; \rho_{1}\right)-\theta\left(x-c(+\infty) t, t ; \rho_{2}\right)\right\|_{L^{1}[0, \infty]} \leq\left|\rho_{1}-\rho_{2}\right| .
$$

In fact, since $c(+\infty)>0$, we have

$$
\lim _{t \rightarrow \infty}\left\|\theta\left(x-c(+\infty) t, t ; \rho_{1}\right)-\theta\left(x-c(+\infty) t, t ; \rho_{2}\right)\right\|_{L^{1}[0, \infty]}=\left|\rho_{1}-\rho_{2}\right| .
$$

Letting $t \rightarrow \infty$ in (5.15), we find that $\left|\rho_{T_{1}}-\rho_{T_{2}}\right|=O(1) e^{-\bar{\sigma} \min \left\{T_{1}, T_{2}\right\}}$. Therefore, the sequence $\left\{\rho_{T}\right\}$ converges to some $\rho_{\infty}$, with

$$
\left|\rho_{T}-\rho_{\infty}\right|=O(1) e^{-\bar{\sigma} T} \text {. }
$$

Combining (5.14), (5.17), and (5.19), we have (5.20)

$$
\left\|u(x, t ; T)-\theta\left(x-c(+\infty) t, t ; \rho_{\infty}\right)\right\|_{L^{\prime}[0, \infty]}=O(1)\left(e^{-\bar{\sigma} T}+T(t-T)^{-1 / 2}\right),
$$

for any $T \leq t$. Setting $T=t^{\varepsilon}$ now gives the result.

\section{THe CASE $c(x)=\tanh (x)$}

In the main case of interest, $c(x)=\tanh (x)$, we can obtain more precise information about the solution. In this case for $\eta=1 / 2$, the coefficient $\eta c^{\prime}+$ $\eta(1-\eta) c^{2}$ is identically one, so that the transformed equation, (4.2) becomes particularly simple.

In the linearly degenerate case, $b=0$, it reduces to a damped heat equation, which admits an exact solution. Transforming back, we find that Green's function for the original (1.1) is just

$$
G(x, t ; y)=\frac{e^{-y}}{e^{y}+e^{-y}} K(x-y-t, t)+\frac{e^{y}}{e^{y}-e^{-y}} K(x-y+t, t),
$$

where $K(x, y)$ denotes the heat kernel. In other words, the fundamental solution is a convex average of dissipation waves moving to the right and to the left, not only time asymptotically, but at each time $t$. We thus see trivially that the solution has all decay and dissipation wave behavior of the heat equation.

For $b \neq 0$, we can treat the term $\left(u^{2}\right)_{x}$ as a quadratic source term and use iteration to show that the solution is bounded by a multiple of the linearly degenerate solution. As in $\S 3$, we can now treat the part of the solution to the right of the origin as a solution of the constant convection Burgers' equation with an exponentially small source term $[(c(x)-c(+\infty)] u)_{x}$ and an exponentially small boundary condition at $x=0$.

Note that, in the linearly degenerate case, the mass of the right dissipation wave can be determined from the initial data by the inner product $\left\langle e^{*}, u_{0}\right\rangle$, with $e^{*}=\frac{e^{y}}{e^{y}+e^{-y}}$. More generally, the dual vector $e^{*}$ is determined by the adjoint equation $-c(x) e_{x}^{*}=e_{x x}^{*}$ with the boundary conditions $e^{*}(-\infty)=0$, 
$e^{*}(+\infty)=1$. Such a solution exists when $c(-\infty)<0<c(+\infty)$ and determines a second quantity that is conserved along with mass.

In the genuinely nonlinear case, the mass of the right diffusion wave again represents a second $L^{1}$ invariant but cannot be calculated explicity. This is analogous to the situation of $N$-waves in hyperbolic systems.

\section{THE INVISCID EQUATION}

Finally, we briefly discuss the associated inviscid equation,

$$
u_{t}+(c(x) u)_{x}+b\left(u^{2}\right)_{x}=0 .
$$

Along characteristics, this becomes

$$
\frac{d u}{d \tau}=-c^{\prime}(x) u,
$$

so that $u$ is decreasing in absolute value.

This allows us to derive a sharp $L^{\infty}$ decay rate, using an argument of Dafermos [1]. Let $\Gamma$ denote the characteristic through $(x, t)$. Then the total mass flux through $\Gamma$ from time 0 to time $t$ is given by the integral of the instantaneous mass flux, $\int_{0}^{t}(f(u)-\lambda u) d t$. Here, $f=c(x) u+b u^{2}$ denotes the flux and $\lambda=c(x)+2 b u$ denotes the characteristic speed, so that the integrand is exactly $-b u^{2}$. Therefore, since $u$ is decreasing along $\Gamma$, the total flux is at least $b u^{2}(x, t) t$.

But, by the $L^{1}$ contraction theorem, the total flux

$$
\left|\int_{-\infty}^{\Gamma} u(\cdot, t) d x-\int_{-\infty}^{\Gamma} u(\cdot, 0) d x\right|
$$

is bounded by $2\left\|u_{0}\right\|_{L^{1}}$. Combining, we have $L^{\infty}$ decay at the rate $t^{-1 / 2}$.

On the other hand, it is not clear that the solution coalesces into two $N$-waves, as happens in the viscous case. In this regard, we remark that the inviscid problem is somewhat in the viscous case. In this regard, we remark that the inviscid problem is somewhat artificial, since it amounts to taking the zeroviscosity limit in one wave family and not in the other.

\section{ACKNOWLEDGMENTS}

The author thanks the referee for several helpful suggestions.

\section{REFERENCES}

1. C. Dafermos, Regularity and large time behavior of solutions of a conservation law without convexity, Proc. Roy. Soc. Edinburgh Sect. A 99 (1985), 201-239.

2. A. Friedman, Partial differential equations of parabolic type, Prentice-Hall, Englewood Cliffs, NJ, 1964.

3. D. Hoff and J. Smoller, Solutions in the large for certain nonlinear parabolic systems, Ann. Inst. Henri Poincaré 3 (1985), 210-220.

4. __ Global existence for systems of parabolic conservation laws in several sapce variables, J. Differential Equations 68 (1987), 213-235.

5. Kruzkov, First order quasilinear equations in several space variables, Math. USSR-Sb. 10 (1970), 112-113.

6. T.-P. Liu, Decay to $N$-waves of solutions of general systems of nonlinear hyperbolic conservation laws, Comm. Pure Appl. Math. 30 (1977), 585-610. 
7. Soc. 56 (1985), 1-108.

8. T.-P. Liu and K. Zumbrun, Nonlinear stability of an undercompressive shock, in preparation.

9. D. Sattinger, On the stability of waves of nonlinear parabolic systems, Adv. Math. 22 (1976), 312-355.

10. K. Zumbrun, B. Plohr, and D. Marchesin, Scattering behavior of transitional shock waves, State Univ. of New York at Stony Brook, Report No. SUNYSB-AMS-91-09, 1991.

Department of Mathematics, Indiana University, Bloomington, Indiana 47405

E-mail address: kzumbrun@indiana.edu 